\title{
Temperature dependence of vortex charges in high-temperature superconductors
}

\author{
Yan Chen, ${ }^{1}$ Z. D. Wang, ${ }^{2,3}$ and C. S. Ting ${ }^{1}$ \\ ${ }^{1}$ Texas Center for Superconductivity and Department of Physics, University of Houston, Houston, Texas 77204, USA \\ ${ }^{2}$ Department of Physics, University of Hong Kong, Pokfulam Road, Hong Kong, China \\ ${ }^{3}$ Department of Material Science and Engineering, University of Science and Technology of China, Hefei 230026, China
}

(Received 25 February 2003; published 3 June 2003)

\begin{abstract}
Using a model Hamiltonian with $d$-wave superconductivity and competing antiferromagnetic (AF) interactions, the temperature $(T)$ dependence of the vortex charge in high- $T_{c}$ superconductors is investigated by numerically solving the Bogoliubov-de Gennes equations. The strength of the induced AF order inside the vortex core is $T$ dependent. The vortex charge could be negative when the AF order with sufficient strength is present at low temperatures. At higher temperatures, the AF order may be completely suppressed and the vortex charge becomes positive. A first-order-like transition in the $T$-dependent vortex charge is seen near the critical temperature $T_{A F}$. For an underdoped sample, the spatial profiles of the induced spin-density wave and the charge-density wave orders could have stripelike structures at $T<T_{s}$, and change to two-dimensional isotropic ones at $T>T_{s}$. As a result, a vortex charge discontinuity occurs at $T_{s}$.
\end{abstract}

DOI: 10.1103/PhysRevB.67.220501

For the past several years, there have been intensive studies on the vortex physics in high-temperature superconductors (HTS). It is well established now that the $d$-wave superconductivity (DSC) with a competing antiferromagnetism (AF) order plays an important role in determining the vortex structure of HTS. Many theoretical studies ${ }^{1-10}$ have shown that the AF order may appear and coexist with the underlying vortices. Experimental facts including neutron-scattering, ${ }^{11}$ moun spin rotation measurement, ${ }^{12}$ and nuclear-magneticresonance (NMR) experiments ${ }^{13,14}$ have provided a strong support to the existence of AF order inside the vortex core in appropriately doped HTS. On the other hand, the electrical charge associated with the vortex in superconductors has also been paid considerable attention both theoretically ${ }^{15-20}$ and experimentally. ${ }^{21,22}$ Based on the BCS theory, Blatter et al. ${ }^{16}$ pointed out that for an $s$-wave superconductor, the vortex charge is proportional to the slope of the density of states at the Fermi level. However, the NMR and nuclear quadrupole resonance (NQR) measurements on $\mathrm{YBa}_{2} \mathrm{Cu}_{3} \mathrm{O}_{7}$ and $\mathrm{YBa}_{2} \mathrm{Cu}_{4} \mathrm{O}_{8}$ (Ref. 21) seemed to obtain results for the vortex charge, contradictory to that predicted from the existing BCS theory with respect to both the sign and the order of magnitude. In addition, if the impact of vortex charge on the mixed state Hall signal is considered, ${ }^{15}$ the sign estimated from Hall effect experiments ${ }^{22}$ for various HTS materials disagrees with the prediction of BCS-type theory. In view of these points, together with the fact that the strong electron correlation with the $d$-wave superconducting pairing was not considered for the vortex charge, we carried out the previous studies $^{20}$ at zero temperature and found that the vortex charge in HTS is strongly influenced by the presence of the induced AF order in the vortex core. The charge carried by a vortex is always positive for a pure $d$-wave superconductor, and it becomes negative when there is sufficient strength of AF order in the vortex core. ${ }^{20}$ Since most relevant experimental phenomena have been observed at finite $T$ not close to zero, including the sign change of the mixed state Hall effect, ${ }^{22}$ it is necessary and valuable to study theoretically the vortex charges at finite $T$, which so far have not been addressed in the literature.
PACS number(s): 74.20.-z, 74.25.Jb

In this paper, we shall investigate the vortex charge as a function of $T$ for both optimally doped and underdoped HTS in detail. Our calculation will be based on the model Hamiltonian on a square lattice with a nearest-neighboring attractive interaction $V_{D S C}$ describing the DSC and an on-site Coulomb repulsion $U$ representing the competing $\mathrm{AF}$ order. We shall adopt a well-developed numerical scheme ${ }^{23}$ to study the vortex structure. Our numerical results at finite $T$ confirm that the sign of the vortex charge is still determined by the AF order in the vortex core, being consistent with our previous work. ${ }^{20}$ The vortex charge could be transformed from negative to positive values as $T$ is increased to a critical value $T_{A F}$ such that the $\mathrm{AF}$ order is completely suppressed at $T>T_{A F}$, and the transition seems to be a first-order-like. For an underdoped sample, we show that the symmetry of the induced spin-density wave (SDW) and the charge-density wave (CDW) may change from stripelike to twodimensional-like at a critical temperature $T_{s}$. A discontinuity of the $T$-dependent vortex charge also appears at $T_{s}$.

The effective mean-field Hamiltonian describing both DSC and SDW orders on a two-dimensional lattice can be represented as

$$
\begin{aligned}
H= & -\sum_{i, j, \sigma} t_{i j} c_{i \sigma}^{\dagger} c_{j \sigma}+\sum_{i, \sigma}\left(U n_{i \bar{\sigma}}-\mu\right) c_{i \sigma}^{\dagger} c_{i \sigma} \\
& +\sum_{i, j}\left(\Delta_{i j} c_{i \uparrow}^{\dagger} c_{j \downarrow}^{\dagger}+\text { H.c. }\right),
\end{aligned}
$$

where $c_{i \sigma}^{\dagger}$ is the electron creation operator and $\mu$ is the chemical potential. In the presence of magnetic field $B$ perpendicular to the plane, the hopping integral can be expressed as $t_{i j}=t_{0} \exp \left[i\left(\pi / \Phi_{0}\right) \int_{\mathbf{r}_{j}}^{\mathbf{r}_{i}} \mathbf{A}(\mathbf{r}) \cdot d \mathbf{r}\right]$ for the nearestneighboring sites $i$ and $j$. A Landau gauge $\mathbf{A}=(-B y, 0,0)$ is chosen. The two possible SDW and DSC orders in cuprates are defined as $\Delta_{i}^{S D W}=U\left\langle c_{i \uparrow}^{\dagger} c_{i \uparrow}-c_{i \downarrow}^{\dagger} c_{i \downarrow}\right\rangle$ and $\Delta_{i j}$ $=V_{D S C}\left\langle c_{i \uparrow} c_{j \downarrow}-c_{i \downarrow} c_{j \uparrow}\right\rangle / 2$, where $U$ and $V_{D S C}$ represent, respectively, the interaction strengths for the two orders. The 
mean-field Hamiltonian (1) can be diagonalized by solving the resulting Bogoliubov-de Gennes equations selfconsistently,

$$
\sum_{j}\left(\begin{array}{cc}
\mathcal{H}_{i j, \sigma} & \Delta_{i j} \\
\Delta_{i j}^{*} & -\mathcal{H}_{i j, \bar{\sigma}}^{*}
\end{array}\right)\left(\begin{array}{c}
u_{j, \sigma}^{n} \\
v_{j, \bar{\sigma}}^{n}
\end{array}\right)=E_{n}\left(\begin{array}{c}
u_{i, \sigma}^{n} \\
v_{i, \bar{\sigma}}^{n}
\end{array}\right),
$$

where the single-particle Hamiltonian $\mathcal{H}_{i j, \sigma}=-t_{i j}$ $+\left(U n_{i \bar{\sigma}}-\mu\right) \delta_{i j}, \quad$ and $\quad n_{i \uparrow}=\Sigma_{n}\left|u_{i \uparrow}^{n}\right|^{2} f\left(E_{n}\right), \quad n_{i \downarrow}=$ $\Sigma_{n}\left|v_{i \downarrow}^{n}\right|^{2}\left[1-f\left(E_{n}\right)\right], \quad$ and $\quad \Delta_{i j}=\left(V_{D S C} / 4\right) \Sigma_{n}\left(u_{i \uparrow}^{n} v_{j \downarrow}^{n *}\right.$ $\left.+v_{i \downarrow}^{*} u_{j \uparrow}^{n}\right) \tanh \left(E_{n} / 2 k_{B} T\right)$, with $f(E)$ as the Fermi distribution function. The DSC order parameter at the $i$ th site is $\Delta_{i}^{D}=\left(\Delta_{i+e_{x}, i}^{D}+\Delta_{i-e_{x}, i}^{D}-\Delta_{i, i+e_{y}}^{D}-\Delta_{i, i-e_{y}}^{D}\right) / 4$, where $\Delta_{i j}^{D}$ $=\Delta_{i j} \exp \left[i\left(\pi / \Phi_{0}\right) \int_{\mathbf{r}_{i}}^{\left(\mathbf{r}_{i}+\mathbf{r}_{j}\right) / 2} \mathbf{A}(\mathbf{r}) \cdot d \mathbf{r}\right]$ and $\mathbf{e}_{x, y}$ denotes the unit vector along $x$ and $y$ directions. In our calculation, the related parameters are chosen as $t=a=1, V_{D S C}=1.2$, the linear dimension of the unit cell of the vortex lattice is $N_{x} \times N_{y}=40$ $\times 20$. This choice corresponds the magnetic field $B \simeq 37 \mathrm{~T}$.

In the absence of the magnetic field and for optimally doped sample $(\delta=0.15)$, the parameters are chosen in such a way that only DSC (with $T_{c} \sim 0.164$ ) prevails in the system. Our numerical results with $U=2.2$ indeed show that the $\mathrm{AF}$ order is induced at low temperature and is suppressed when $T$ is larger. The spatial profiles of the superconductivity order parameter, staggered magnetization, and the charge density near the vortex core are plotted at two different temperatures in Fig. 1. Here, the staggered magnetization of the induced $\mathrm{AF}$ or SDW order is defined as $M_{s}^{i}=(-1)^{i} \Delta_{i}^{S D W} / U$. The $\mathrm{AF}$ order is generated in the region where the DSC order parameter is partially suppressed. The low-temperature results at $T=0.02$ are shown in Figs. 1(a) to 1(c), where the AF order is nucleated and spreads out from the core center, and the vortex charge is negative. The induced $\mathrm{AF}$ order behaves similar to a two-dimensional SDW with the same wavelength in the $x$ and $y$ directions. The higher-temperature results at $T=0.05$ are shown in Figs. 1(d) to 1(f), where the AF order is completely suppressed and the vortex charge becomes positive. The appearance of the SDW order pinned by the vortex lattice at low $T$ strongly enhances the net electron density (or depletion of the hole density) at the vortex core as shown in Fig. 1(c). The present result is consistent with that of Ref. 20 where the doping and $U$-value dependences of vortex charges are examined. Both works reach the same conclusion: the vortex charge is negative when a sufficient strength of SDW order is induced, while it becomes positive with the suppression of the SDW order due to increasing the $T$ or doping level, or decreasing the $U$ value.

Figure 2(a) plots the phase diagram of $T$ versus $U$ for positively (hole-rich) and negatively (electron-rich) charged vortices. It is obvious that the $\mathrm{AF}$ vortex core can easily be induced in the low-temperature regime or with a stronger $\mathrm{AF}$ interaction $U$, while the pure DSC core tends to exist in the high $T$ or smaller $U$. The electron density inside the core is higher than the average density in the low-temperature region, while the electron density becomes lower than the average in the high-temperature region. There exists a clear boundary between these two phases. To estimate the vortex
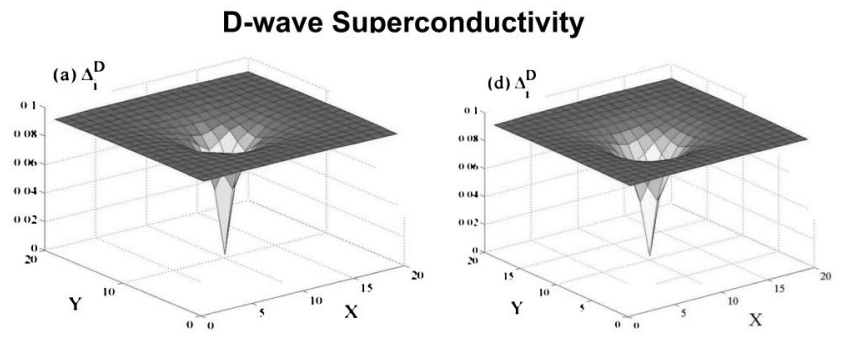

Staggered Magnetization
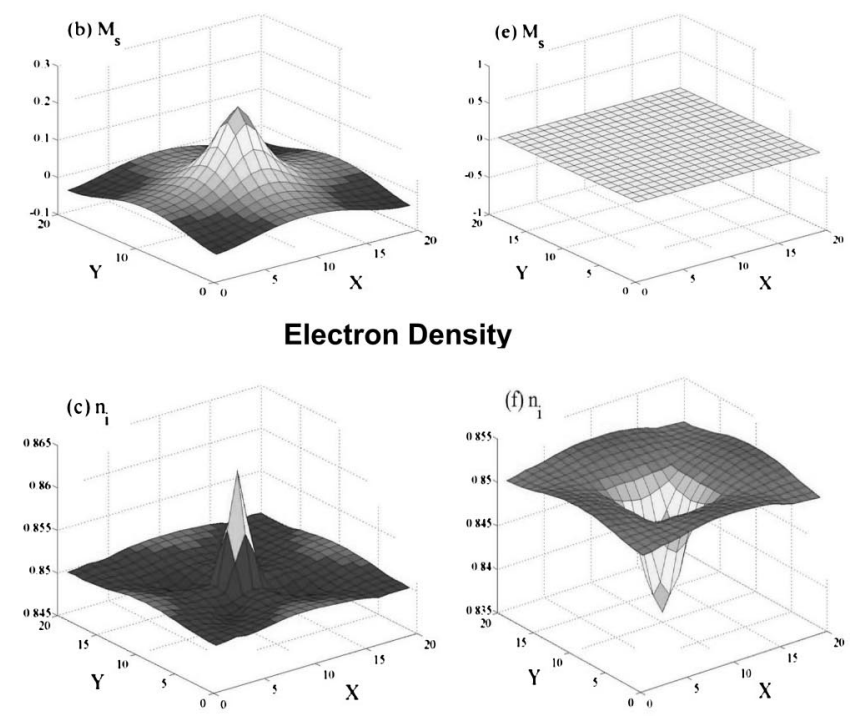

FIG. 1. Spatial variations of the DSC order parameter $\Delta_{i}^{D}[(\mathrm{a})$ and (d)], staggered magnetization $M_{i}^{s}$ [(b) and (e)], and net electron density $n_{i}[$ (c) and (f)] in a $20 \times 20$ lattice. The left panels [(a)-(c)] and the right panels $[(\mathrm{d})-(\mathrm{f})]$ are for $T=0.02$ and $T=0.05$, respectively. The averaged electron density is fixed at $\bar{n}=0.85$ and $U=2.2$.

charge, we first determine the vortex size by examining the spatial profile of DSC order parameter. Next, we sum over the net electron density inside the vortex core. In Fig. 2(b), we depict the chemical potential versus $T$ at a fixed doping level ( $\delta=0.15$ with $U=2.4$ and $U=2.2$ ). The result (solid line) for $U=2.4$ exhibits a first-order-like transition at $T_{A F}$ $\sim 0.077$, indicating the existence of induced AF order below this critical temperature. For $U=2.2$, the result (dashed line)
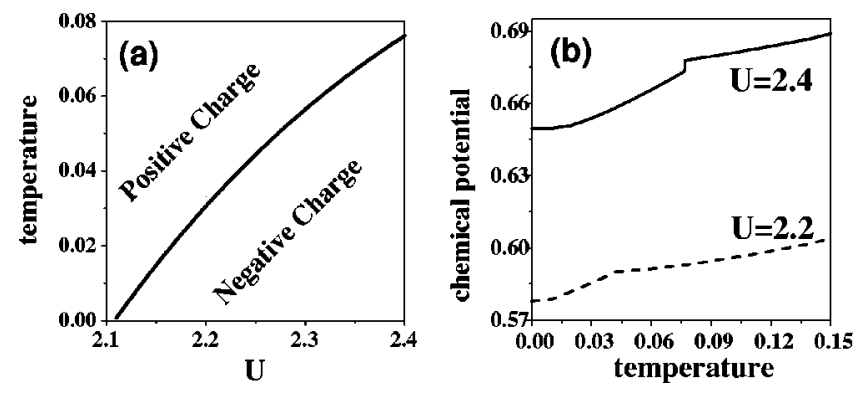

FIG. 2. (a) Phase diagram of interaction strength $U$ versus temperature $T$ for positively and negatively charged vortices at optimal doping $\delta=0.15$. (b) Temperature dependence of chemical potential for $U=2.4$ and $U=2.2$. 


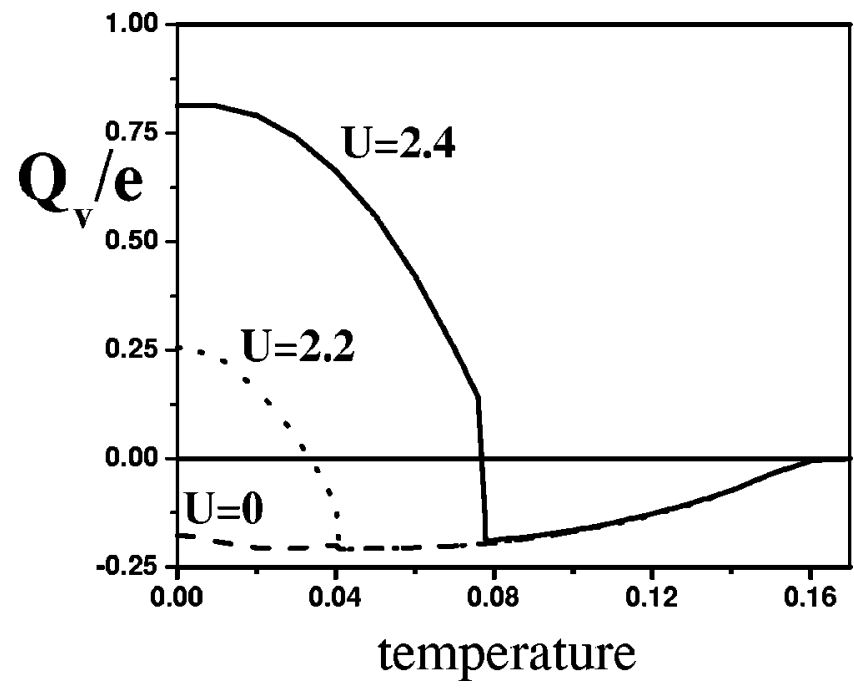

FIG. 3. The temperature dependence of the number of vortex charge $Q_{v} / e$ for $U=2.4$ (solid line), $U=2.2$ (dotted line), and $U=0$ (dashed line). The averaged electron density is fixed at $\bar{n}=0.85$.

seems to show a very weak discontinuity which can hardly be seen in Fig. 2(b). The above finding may be closely related to the experimental results for underdoped $\mathrm{TlBa}_{2} \mathrm{Cu}_{4} \mathrm{O}_{8}$ (Ref. 14) where the vortex core with induced AF order below $T_{A F}$ was reported. Also from Fig. 2(b), a discontinuity in the slope of the $T$-dependent chemical potential at $T_{A F}$ for both cases can be clearly observed. We expect that the local density of states (LDOS) of the vortex core should have double peaks $5,7,10,25,26$ near zero bias for $T<T_{A F}$; while for $T$ $>T_{A F}$ only a single broad zero-bias peak can appear in the LDOS as one of the essential characteristics of DSC. ${ }^{23}$ It would be interesting to measure this temperature evolution in the LDOS by future scanning tunnel microscopy (STM) experiments. Also, interestingly, even though the origin of the Hall sign anomaly in HTS is still debatable, ${ }^{24}$ the vortex charge could make an additional contribution to the sign change in the mixed state Hall conductivity. ${ }^{15}$ As a result, we predict that a sign reversal in the Hall signal may occur at $T$ not too far below $T_{A F}$.

In Fig. 3, we plot the vortex charge number $Q_{v} / e$ as a function of $T$ for three typical $U$ values, where $e<0$ is the charge of an electron. The $T$ dependence of the induced staggered magnetization $M_{s}^{c}$ at the vortex core center has also been examined in previous studies ${ }^{27,28}$ where the induced AF order is shown to disappear for $T>T_{A F}$. From Fig. 3 and for $U=2.4$, one can clearly see an abrupt jump in the number of vortex charge $Q_{v} / e$ as $T$ varies around $T_{A F} \sim 0.077$, which is consistent with the critical temperature as shown in Fig. 2(b). This positive to negative vortex charge transition seems to be first-order-like. The magnitude of the discontinuity reduces to one-third when $U=2.2$ at $T_{A F} \sim 0.04$. Here, the discontinuity occurs at the positive to positive vortex charge transition, not the positive to negative transition as studied in the case of $U=2.4$. This is because slightly below $T_{A F}$, the induced AF order is too weak to make the vortex charge negative. For a pure DSC case with $U=0$, the vortex charge $Q_{v}$ is always positive and its magnitude first increases

\section{Staggered magnetization}

Electron density
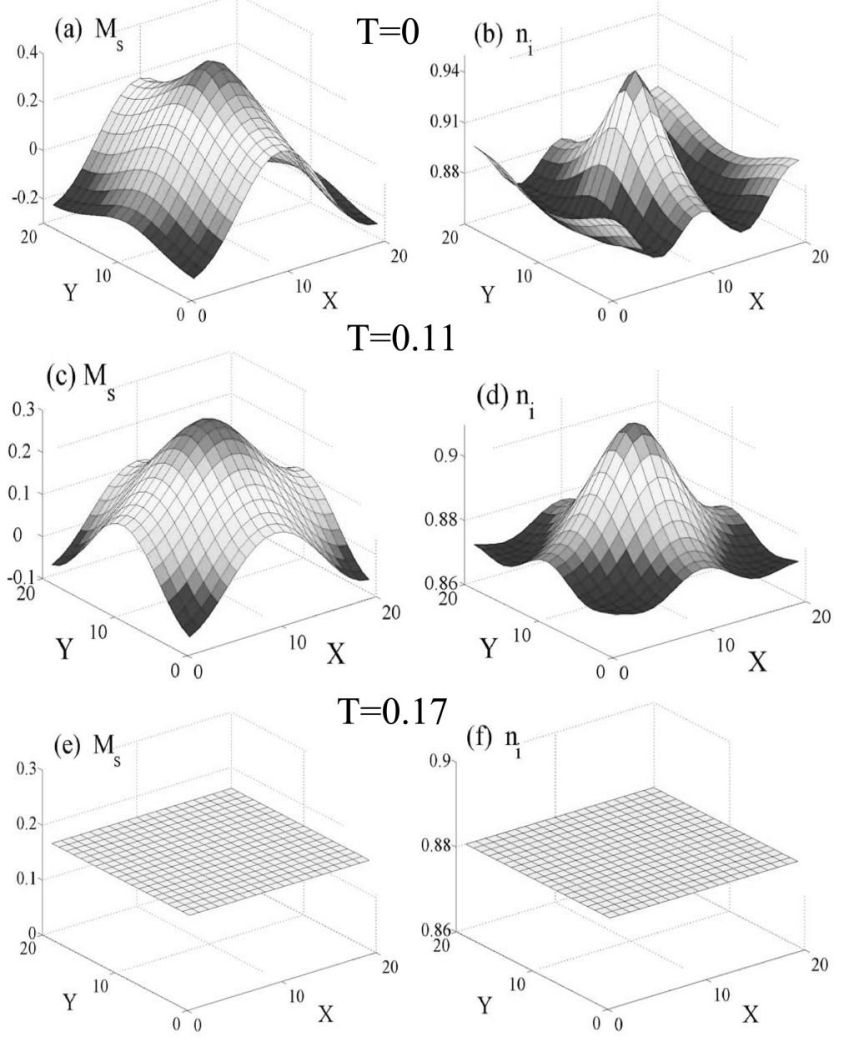

FIG. 4. Spatial variations of the local electron density $n_{i}$ for various temperatures (a) $T=0.0$, (b) $T=0.11$, and (c) $T=0.17$. The strength of the on-site repulsion $U=2.4$. The averaged electron density is fixed at $\bar{n}=0.88$.

slightly with $T$ and then decreases to zero as $T$ approaches to $T_{c}$. The critical temperature $T_{A F}$ is $U$-value dependent or sample dependent. The larger $U$ corresponds to larger $T_{A F}$. These results confirm that the vortex charge is strongly influenced by two competing effects: the suppression of the DSC order at the core center leading to the depletion of electrons, and the induction of the AF order which favors the accumulation of electrons. The condition for the negative vortex charge to appear depends solely on whether there is sufficient AF order inside the vortex core. We emphasize that our results are robust and indifferent to band parameters, and should give a qualitative description on the vortex physics in HTS.

In the following, we are going to investigate the temperature dependence of the vortex charge for the underdoped HTS ( $\delta=0.12$ ). For $U=2.4$, the spatial profiles of the staggered magnetization and electron density near the vortex core at three different temperatures are plotted in Fig. 4. As discussed in our previous work, ${ }^{7}$ the parameters used to obtain Fig. 4 yield stripelike structures in the DSC, SDW, and CDW order parameters at very low-temperature. In the present work, the DSC and SDW orders coexist in underdoped sample below $T_{c}$, which implies $T_{A F}>T_{c}$. So the question arises: will these stripelike structures persist with increasing temperature? From our calculations, the spatial profiles of these order parameters may go through three dif- 


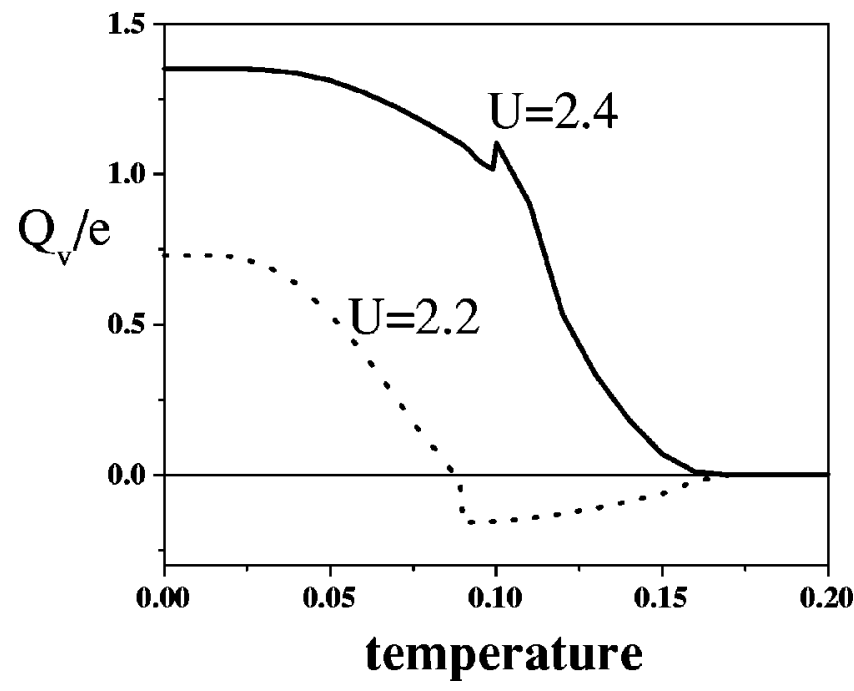

FIG. 5. The vortex charge number $Q_{v} / e$ for $U=2.4$ (solid line) and $U=2.2$ (dotted line) as a function of temperature $T$. The averaged electron density is fixed at $\bar{n}=0.88$.

ferent symmetries as $T$ increases. At $T=0$, the spatial distribution of SDW $\left(M_{s}\right)$ and $\operatorname{CDW}\left(n_{i}\right)$ orders exhibit quasione-dimensional stripelike behavior [see Figs. 4(a) and 4(b)]. When $T$ is raised to $T=0.11$ above a characteristic temperature $T_{s}(\sim 0.1)$, the symmetry of the patterns change to isotropic and two dimensional [see Figs. 4(c) and 4(d)]. Above $T_{c}(\sim 0.16)$ and at $T=0.17$, the residual $\mathrm{AF}$ and electron density become uniform, as shown in Figs. 4(e) and 4(f). The AF order vanishes when $T>T_{A F}$.

Finally, we display the temperature evolution of the vortex charge number $Q_{v} / e$ for the underdoped case $\delta=0.12$ with $U=2.4$ and $U=2.2$ in Fig. 5. Comparing with Fig. 3, the magnitudes of vortex charges for both cases in Fig. 5 at low-temperatures become larger as a result of stronger AF order near the vortex core in underdoped samples. For $U$ $=2.4$, the $Q_{v} / e$ versus temperature curve (see the solid line in Fig. 5) exhibits a discontinuity at $T_{s} \sim 0.1$, which reflects the characteristic temperature between two different symmetries. For $U=2.2$, the spatial distribution patterns for SDW and CDW are always isotropic and two-dimensional with $T_{A F} \sim 0.09$, where an appreciable discontinuity in $Q_{v} / e$ (see the dotted line in Fig. 5) is present.

Recently, STM experiments ${ }^{29,30}$ showed the existence of inhomogeneities in the electron density even for the optimally doped $\mathrm{Bi}_{2} \mathrm{Sr}_{2} \mathrm{CaCu}_{2} \mathrm{O}_{8+x}$, there the hole density varies from 0.1 to 0.2 . From our previous paper ${ }^{20}$ and the present study, we found that there may exist two types of vortex cores, either negatively charged or positively charged. By increasing $T$, the number of negatively charged vortices decreases, while that of positively charged vortices increases. This may have a profound effect on the mixed state transport properties in HTS. We have also examined the effect of the long-range Coulomb interaction on the charge distributions using the Poisson equation. Our results show that its influence on the vortex charge is weak and there is no qualitative change in our conclusion. Finally, we wish to point out that our numerical study has been performed in a strong magnetic field. So far we are not able to extend this calculation to a much weaker field. However, according to the extrapolation scenario, ${ }^{20}$ the magnitude of the induced AF order follows roughly a linear relationship with $B$. The strength of the AF order and the magnitude of vortex charge are estimated to be much smaller as $B$ is reduced to the experimental value $(\sim 9.4 \mathrm{~T})$. Despite this difficulty, our numerical approach should give a consistent description to the behavior of vortex charges in HTS.

We are grateful to Professor S. H. Pan, B. Friedman, and Dr. J. X. Zhu for useful discussions. This work was supported by the Robert A. Welch Foundation and by the Texas Center for Superconductivity at the University of Houston through the State of Texas. Z.D.W. also appreciates the support from the RGC grant of Hong Kong (Grant No. HKU7092/01P), the Ministry of Science and Technology of China under Grant No. 1999064602, and the URC fund of HKU.
${ }^{1}$ D.P. Arovas et al., Phys. Rev. Lett. 79, 2871 (1997).

${ }^{2}$ N. Ogata, Int. J. Mod. Phys. B 13, 3560 (1999).

${ }^{3}$ E. Demler et al., Phys. Rev. Lett. 87, 067202 (2001).

${ }^{4}$ M. Ichioka et al., J. Phys. Soc. Jpn. 70, 33 (2001).

${ }^{5}$ Jian-Xin Zhu, and C.S. Ting, Phys. Rev. Lett. 87, 147002 (2001).

${ }^{6}$ H.D. Chen et al., Phys. Rev. Lett. 89, 137004 (2002).

${ }^{7}$ Yan Chen and C.S. Ting, Phys. Rev. B 65, 180513(R) (2002).

${ }^{8}$ Yan Chen et al., Phys. Rev. B 66, 104501 (2002).

${ }^{9}$ M. Franz et al., Phys. Rev. Lett. 88, 257005 (2002).

${ }^{10}$ A. Ghosal et al., Phys. Rev. B 66, 214502 (2002).

${ }^{11}$ B. Lake et al., Science 291, 1759 (2001); B. Lake et al., cond-mat/0104026 (unpublished).

${ }^{12}$ R.I. Miller et al., Phys. Rev. Lett. 88, 137002 (2002).

${ }^{13}$ V.F. Mitrovic et al., Nature (London) 413, 501 (2001); cond-mat/0202368, Phys. Rev. B (to be published 1 June 2003).

${ }^{14}$ K. Kakuyanagi, K. Kumagai, Y. Matsuda, and M. Hasegawa, cond-mat/0206362 (unpublished).
${ }^{15}$ D.I. Khomskii and A. Freimuth, Phys. Rev. Lett. 75, 1384 (1995).

${ }^{16}$ G. Blatter et al., Phys. Rev. Lett. 77, 566 (1996).

${ }^{17}$ N. Hayashi et al., J. Phys. Soc. Jpn. 67, 3368 (1998).

${ }^{18}$ J. Kolacek et al., Phys. Rev. Lett. 86, 312 (2001).

${ }^{19}$ P. Lipavsky et al., Phys. Rev. B 66, 134525 (2002).

${ }^{20}$ Yan Chen et al., Phys. Rev. Lett. 89, 217001 (2002).

${ }^{21}$ K. Kumagai et al., Phys. Rev. B 63, 144502 (2001).

${ }^{22}$ T. Nagaoka et al., Phys. Rev. Lett. 80, 3594 (1998).

${ }^{23}$ Y. Wang and A.H. MacDonald, Phys. Rev. B 52, R3876 (1995).

${ }^{24}$ Z.D. Wang et al., Phys. Rev. Lett. 72, 3875 (1994).

${ }^{25}$ Ch. Renner et al., Phys. Rev. Lett. 80, 3606 (1998).

${ }^{26}$ S.H. Pan et al., Phys. Rev. Lett. 85, 1536 (2000).

${ }^{27}$ M. Takigawa et al., Phys. Rev. Lett. 90, 047001 (2003).

${ }^{28}$ Yan Chen, Jian-Xin Zhu, and C.S. Ting, cond-mat/0302114 (unpublished).

${ }^{29}$ S.H. Pan et al., Nature (London) 413, 282 (2001).

${ }^{30}$ K.M. Lang et al., Nature (London) 415, 412 (2002). 